\title{
Congestion Control in VANET at MAC Layer: A Review
}

\author{
Kanchan Nahar \\ Computer Science Engineering, \\ L.R. Engineering \& Technology, \\ Solan, India
}

\author{
Swati Sharma \\ Computer Science Engineering \\ Punjab Engineering College, \\ Chandigarh, India
}

\begin{abstract}
Vehicular ad-hoc network (VANET) has become an encouraging area in the research environment. Intelligent transport system (ITS) allows VANET, to improving traffic efficiency and management $\&$ driver safety. For better communication VANET falls under two categories such as vehicle-tovehicle $(\mathrm{V} 2 \mathrm{~V})$ \& Vehicle-to-Infrastructure, under the wireless network environment. Every vehicle moving fast and also exchange their information to the immediate vehicle, therefore, the system is loss packet sometimes and high density of vehicles cause to the problem of congestion. Medium Access Control (MAC) plays a vital role to boost the real-time communication within vehicles and roadside components with limited transmission collision. Due to the VANET properties, managing communication at the MAC layer are a serious issue in the collision control scheme. Therefore, it results in packet loss and end-toend delay during communication. The European Telecommunication Standard Institute (ETSI) designed the Decentralized Congestion Control (DCC) mechanism, to handle these kinds of crucial challenges. This paper represents the several characteristics of VANET along with their key challenges associated with congestion control. Besides, the obtainable solutions are represented in detail along with their barriers. This paper focuses on maintaining the channel load measured by the DCC mechanism using various transmitted parameters such as rate, data-rate, sensitive, access control, and power transmission.
\end{abstract}

\section{Keywords-VANET, ITS, congestion control, MAC, DCC}

\section{INTRODUCTION}

Now a day's intelligent transport system (ITS) has grown rapidly as the purpose of improving traffic efficiency and management. ITS provide Vehicular ad-hoc network (VANET), in order to define routes, transport, reliable transmission efficient channel access mechanism. In VANET, vehicles are communicated through On-Board Unit (OBUs) and the Authenticating Unit (AU). VANET provides two types of communication that are vehicle-to-vehicle (V2V) and vehicle-to-infrastructure (V2I) Infrastructure is cost effective; therefore $\mathrm{V} 2 \mathrm{~V}$ communication is more efficient than V2I. VANET has two type of applications namely safety, and non-safety application. Safety application is very crucial as it safe the life. For VANET, Dedicated Short-Range Communication (DSRC) has been developed in the 1990s.

To permit the communication in VANET, IEEE $802.11 \mathrm{p}$ is released that combines physical (PHY) and MAC layer specification. IEEE 802.11p PHY layer is a modified class of the 802.11a specification. It adopts orthogonal frequency-division multiplexing (OFDM) with $10 \mathrm{MHz}$ channel and uses data rates varying from $3 \mathrm{Mbps}-27 \mathrm{Mbps}$. MAC layer uses the enhanced distributed channel access (EDCA) which is based on the carrier sense multiple access with collision avoidance (CSMA/CA) protocol and prioritization. For reliable communication, the protocol stack with the IEEE 1609 WAVE family is defined. For the multi-channel environment, the MAC layer is correctly modified. These EDCA functions help to handle the queues for packets dissemination to the vehicular environment. WAVE support the IPv6 protocol stack as well as handle the WAVE-mode short message protocol (WSMP), carry the high-priority message for the sensitive safety. VANET possesses many applications on the road. These applications can be divided into two subcategories that are safety application and non-safety application. Safety applications are lane change warning, post-crash warning, navigation, Low Bridge warning etc. Non-safety applications are weather information, internet access, toll tax and parking availability etc. Safety application can help to reduce the number of accidents. $60 \%$ of accidents should be avoided if the driver receives a warning message, at least one-half second before the accident. Safety messages include the two types for communication beacon message and eventdriven message. Beacon message used for status of a vehicle, for example; location, speed etc. The event-driven message used for warning messages, for example; crash warning, children warning etc.

Congestion is the main issue on the MAC layer. Congestion occurs when a vehicle sends data against the router capacity such as slow hosts, limited bandwidth and data arriving from multiple lines at the same time. The system is not balanced such as correcting the problem at one router will probably just move the bottleneck to another router. Incoming messages placed in queues such as long queue delay will cause a packet to be resent; the overflowing queue will cause a packet to be dropped. However, in VANET, congestion occurs when the packets are increased over the capacity of the network and it results in packets loss, quality of services degradation, etc. However, the MAC layer plays a vital role in controlling congestion.

Therefore, congestion control schemes are analyzed based on reliability, scalability and throughput of the congested network. IEEE 802.11p based congestion control approaches aim to overcome the channel load and provide fair communication between vehicles. In general, to prevent the congestion occurrence we categorized the congestion control mechanism based on time-turning the transmission of parameters is called proactive, reactive and hybrid congestion control.

The remainder of the paper is structured as follows. Section II summarizes the characteristics, applications, VANET issues and protocol stack of vehicular communication. Section III provides an overview of congestion control. Section IV analyzes congestion control mechanism. Finally, section $\mathrm{V}$ concludes the paper. 


\section{BACKGROUND}

In this section, firstly we discuss the various characteristics and then proceed to the various applications and the challenging issues of VANET. Lastly, comparative analysis of the protocols stacks uses for reliable communication by VANET.

\section{A. Characteristics of VANET}

VANET is a special type of ad-hoc network. Thus enable a various application for moving vehicles. Mobility of vehicles posse various unique characteristics are as follows.

- High mobility: Nodes are not fixed in VANET, usually, they are moving fast. This making too hard to predict the node's position and also made difficult to maintain the privacy of node.

- Network topology: Vehicles are not fixed but the network is fixed. Vehicles are changing their speed these make changing in position on the road.

- Unbound network size: VANET can be executed into a city, more than one city, several countries these result shows that VANET's network size is geographical boundless.

- Frequently Exchange information: Information is gathered using the ad-hoc network. In this, fairly aim to collect all the information from to nodes and RSU. Here beacon plays a role to exchange information among the nodes.

- Wireless communication: For better communication among users, VANET uses electromagnetic waves.

\section{B. Applications of VANET}

VANET's applications play a vital role in communication among vehicles for controlling safety. Mainly, there are two types of applications such as safety related and non-safety related. These are explained in detailed manner.

1) Safety-related application: This application gives a warning message for public safety.

a) Intersection collision warning: Collect information about road intersection.

b) Curve speed warning: This indicates the dissemination of message for the high speed approaching vehicles towards the curves to prevent accidents.

c) Traffic optimized: Traffic can be optimized by the use of sending signals to avoid jam, accidents etc. Among the vehicles, it results in vehicles chose their alternate path at the same time.

d) Emergency electronic brake lights: Warn other vehicles for sudden hard braking.

e) Cooperative adaptive cruise control: Maintain the vehicle's speed at the road.

f) Low bridge warning: It indicates alert message for the driver to know about the height and width of the bridge.

2) Non-Safety related application: These applications provide services to the user. We discuss the following service to the user apart from a safety view. a) Peer to peer application: These applications are helpful to pair or connect with the other nodes in the network for file sharing, example music, and movie, file sharing etc.

b) Internet connection: It maintains an internet connection with vehicles all the time.

c) Other services: VANET has other several applications for a user such as toll taxes, parking place, fuel station, restaurants etc.

\section{Challenging issues in VANET}

The highly dynamic topology and frequent disconnections between vehicles give rise to various challenges. Some of them are described below.

- Security Issue: For the life-critical application, the message should be transmitting it fast. Sometimes participant node performs various activities therefore, they disturbed the network. Example; Denial of service.

- Technical Issue: Due to the high density, channel load and network topology change frequently, the management of the network is difficult. The use of the electromagnetic wave for communication might affect the environment. In the MAC layer, technical issues came at design and architecture level.

- High Bandwidth: Infotainment application, wants streaming data at high quality. Example; 3D maps and navigation require automatic update frequently.

- Congestion: In IEEE 802.11p as the vehicle density increases, the contention for channel usage also increases. This lead to the collision of messages decreases throughput and causes congestion among vehicle for the channel access.

\section{Protocol stack}

The protocol stack of vehicular communication used by USA (WAVE) and Europe (DSRC) is described in detail.

\section{1) Wireless Access in a Vehicular Environment (WAVE)}

A lot of research conducted around the world to help define the standard for the vehicles ad-hoc network that fairly works on routing algorithms, frequency allocation, security issues, PHY and link layer standard, and some new application. VANET used WAVE for communication. WAVE consist the IEEE 1609.x family as shown in Fig. 1.

These family members are:

- IEEE 1609.1 : WAVE Resource Manager Application, define service for vehicle

- IEEE 1609.2 : Wave Security Application, defines secure message

- $\quad$ IEEE 1609.3 : WAVE data Exchange, using routing the message between network layer and transport layer

- IEEE 1609.4 : WAVE Multi-hops operation, based on IEEE 802.11 that specify PHY and MAC layer 


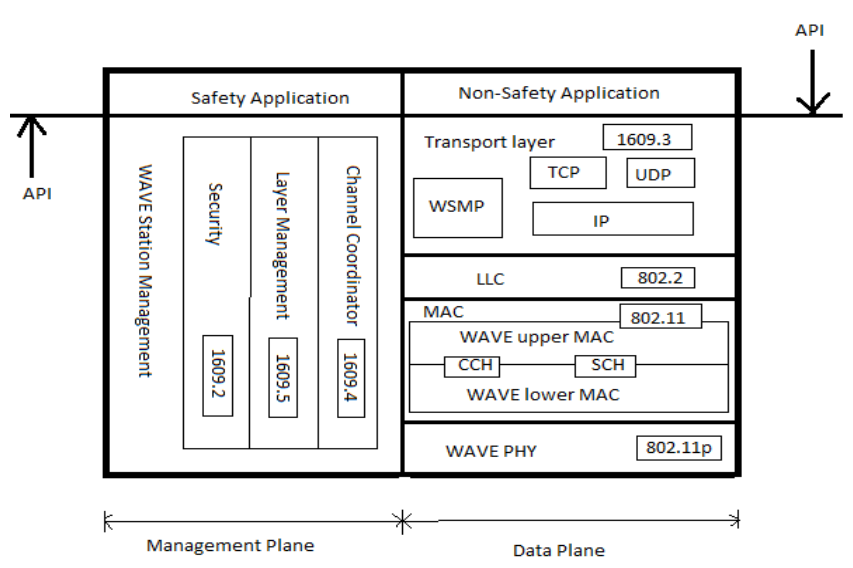

Fig 1: WAVE protocol stack

\section{2) Dedicated Short Range Communication (DSRC)}

DSRC is a wireless communication channel, it could be established the communication connection by one-way or two-way. It directly communicates with vehicles automatically from short-range to medium-range. DSRC band operate the IEEE802.11 devices for $\mathrm{v} 2 \mathrm{x}$ communication. DSRC spectrum using $5.9 \mathrm{GHz}$ bandwidth for reliable communication however partitioned into seven of $10 \mathrm{MHz}$ wide channels. Here only one control channels $(\mathrm{CCH})$ i.e. 178 channel $(5.885-5.895 \mathrm{GHz})$, it is mainly used for the safety communications (as shown in Fig. 2). For the future safety application purpose, two channels are reserved in WAVE. The idle channels are known as a service channel (SCH), which is worked for both safety and non-safety applications. At the PHY level, the IEEE802.11p design to make a fair communication connection with WAVE devices among the fast-moving vehicle in the environment.

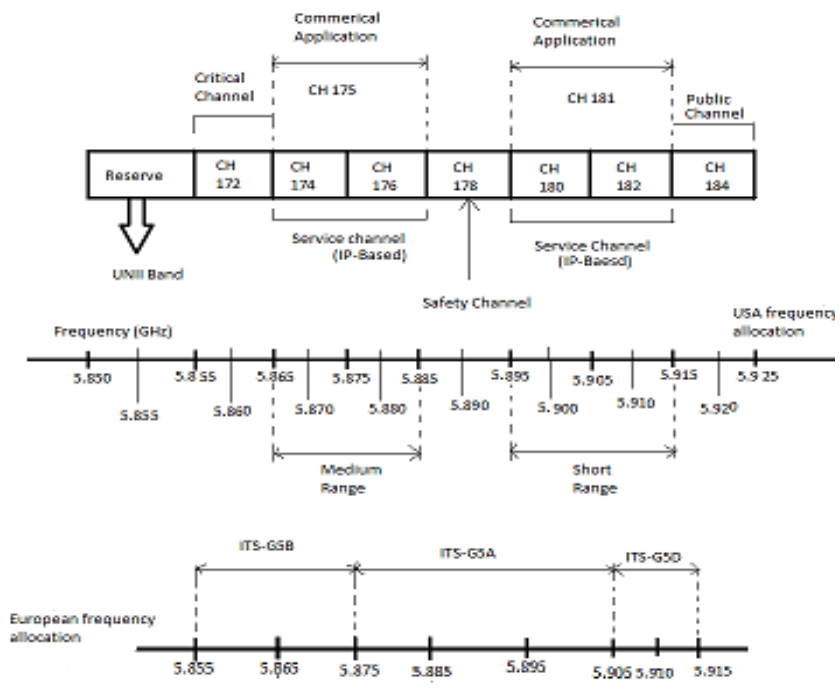

Fig 2: DSRC spectrum

\section{CONGESTION CONTROL}

Intelligent Transport System (ITS) is designed by ETSI. When vehicle density increases MAC layer suffer from congestion due to increase on channel load and this situation decrease the transmission of safety message within the predefined deadline. To the reduce channel load ETSI design the Decentralized Congestion Control (DCC) mechanism that adapts various transmission parameters.
- DCC Access: to control congestion by an act on transmission parameters (Transmit power control (TPC), Transmit rate control (TRC), Transmit data rate control (TDC), DCC Sensitivity control (DSC), Transmit access control (TAC).

- DCC Net: mapping the traffic with Cooperative Awareness Message (CAM) to DCC profile.

- DCC facilities: provide service according to Decentralized Environment Notification Message (DENM) and CAM profiles.

- DCC Management: inter-operation between the different layer-specific DCC entities.

ETSI has been defined two types of messages that are CAM and DENM. CAM provides information of a vehicle presence, position and basic status to one-hop neighbour within 1 to $10 \mathrm{~Hz}$ range of frequency. DENM simulated to an eventdriven message. The message is triggered when ITS station detects any kind of hazard event, it broadcasting the DENM message to the specific geographical area repeatedly, till the event is over. The complete mechanism of DCC is shown in Fig. 3.

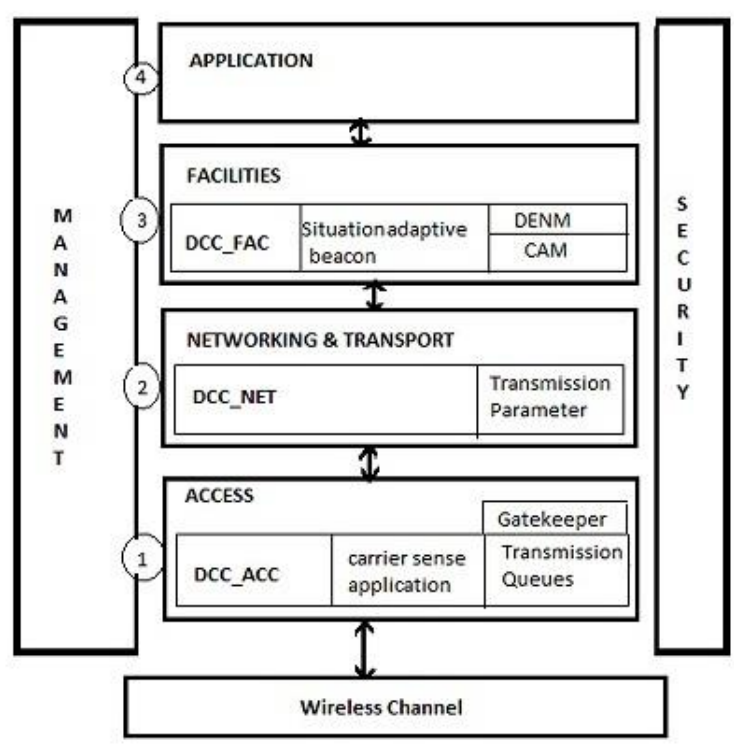

Fig3: DCC mechanism

\section{CONGESTION CONTROL CLASSIFICATION}

Congestion is a big problem in the data network, and control congestion is more challenging because of the highdensity environment and frequent changes in network topologies. Congestion control schemes are focused to provide the fair and reliable channel access. However, it uses the different transmission parameters with a predefined threshold value, to reduce the channel load. This section describes the various congestion control category available in literature along with the finding of research gaps such as reactive, proactive and hybrid.

\section{A. Reactive congestion control}

Reactive congestion control directly works on the channel load to overcome the congestion in vehicular network. This approach takes an action after the congestion is occurred. It also helps to improve the congestion situation to normal con- 
ditions. Packet generation and fairness both are the major characteristics for reducing the congestion in a vehicular network. Main disadvantage of this approach it increases latency.

Subramanian et al. showed that a guard is around transmitters, therefore responsible for the minimum performance of IEEE802.11p MAC at high density. Poor packet reception rate depends upon the ALOHA behaviour. To reduce the channel load ETSI developed the DCC. DCC is an asynchronous algorithm; however, it uses different parameters for transmission. At various congestion conditions, parameters of transmission don't modify easily. Thus the author aims to minimize this problem by using transmission of power control (TPC) algorithm. TPC allows the dissimilar to transmit power values at different channel load. Due to the high network flow, congestion is disturbing the vehicular network. As in result packet is a loss, decrease the throughput and also energy was wasted. To improve the packet delay ratio and quality of service, Wang et al. purposed the node prioritybased congestion control protocol (PCCP). To calculate the degree of a collision, they measure the packet inter-arrival time along with the time of packet service. Also, maintain the degree of congestion by using hop-by-hop control as the priority of node index. PCCP schemes reduced the collision every efficiently and faster, instead of other energy efficiency techniques.

Bansal et al. proposed an error model based on adaptive rate control (EMBARC) to control the saturated condition in a vehicular network. EMBARC is an extension of LIMERIC. To trace the error in a congested network, EMBARC uses the adaptive rate control. Here pre-emptive scheduling is using in tracking techniques. This protocol aims to reduce the channel load for the high-density vehicle. Hence EMBARC mechanism is best tracking accuracy between the wide ranges of vehicle densities. Smai et al. uses timeout mechanism to find the congestion in-network, and also use the handshaking in the router, therefore help to inform the congestion area. This mechanism uses the specific area of the network interface and the processing elements. Performance is evaluated by the network stability. Therefore it tries delaying in worst-case and providing the bounds on average delay, it might be possible by to choosing the right timeout.

For a high-speed network, Liu et al. purposed a novel congestion control mechanism called TCP-Illinois. To control and manage the congestion, TCP-New Reno, SACK TCP and TCP-Reno are the traditional versions of TCP congestion control schemes. This mechanism is truly based on widow size (increased or decreased). This algorithm using the information about packet loss to diagnose the size of the window should be increased or decreased and also use the knowledge about queuing delay to decide the load of increment or decrement. Hence TCP-Illinois helps to attain maximum throughput, assign the network resource fairly. TCP-Illinois also another quality to improve the robustness against sudden variation in delay measurements, busty packet arrival process.

Mondal et al. present the work to control the congestion dynamically along with the reducing transmission rate. The rate transmission to control the messages among the vehicles, only the authorized vehicles can utilize the resources in the vehicular network. To protect the vehicular network from the unwanted message, at the root level certifying authority (CA) have been used, at a middle level, the base stations are used, and at leaf level vehicles are used. Hence each vehicle controls the triggering of the unsafe message in VANET.

Congestion in the vehicular network is a big issue. To label this issue, Benatia et al. designed a novel Markov chain model. However, this model has followed the four tread: congestion detection phase, beacon transmission rate, buffer monitoring and priority assignment to simplify the emergency packets dissemination. In vehicular network, the beacon consuming the large amount the bandwidth, therefore it results in event-driven warning message are not properly transmit in a vehicular network.

\section{1) Observation:}

Reactive congestion control approaches come under the closed

loop congestion control. These protocols are used to control the congestion after it occurs. This congestion control category adjusts either the transmission of power or packet-rate generation measured based on channel load. Moreover, to achieve the maximum fairness, vehicle exchanges their local measurements with immediate neighbour's vehicle. The channel load ratio is calculated as the amount of time during which the channel was busy. These approaches are not able to avoid the collision on the wireless medium and they are not supports to different classes of messages of prioritization.

\section{B. Proactive congestion control}

Proactive congestion control is the second class of congestion control mechanism. These algorithms use the modelbased protocols, based on knowledge such as several vehicles in the area and data packet generation behaviour. However, it tries to estimate which transmit parameter will not come under in saturated condition while providing the necessary application-level performance at the same time. In particular, these mechanisms utilize a system model to evaluate the channel load under the defined parameters of transmission and optimization algorithms also determine maximum transmit power and/or rate.

In a vehicular environment, for safety applications of communications is primarily used, radio performance might be critically threatened by congestion control. The big advantage of this approach is lower message latency (router are immediately available). While the communication model and accurately estimate is a drawback in a vehicular environment. Yang et al. proposed the vehicle-to-vehicle communication protocol mechanism for cooperative collision warning (VCCW). VCCW prevents congestion during the packet generation rate and also achieve delivery of emergency messages at low-latency. VCCW works as an open-loop controller. However, when a vehicle comes at an abnormal state, the vehicle controller monitors the vehicle dynamically and activates the collision warning automatically. Performance is predicted by only the packet generation rate. To achieve network stability channel feedback plays a role. To use of primary feedback, transmission rate might be adjusted easily, also is helping to decide emergency.

Wischhof et al. designed a hop-by-hop proactive congestion control mechanism for the vehicular network. This congestion control mechanism truly based on packet forwarding 
and utility function. This mechanism encrypts the related information in a data packet; however, is transmitted data transparently. A decentralized scheme helps to calculate the "average utility value" of each node according to the utility of its data packets. Thus the part of accepting data rate will be share to its relative priority. For calculating the performance of a network, purposed decentralized utility-based packet forwarding and congestion control (UBPFCC) is implemented at topmost of the IEEE802.11 MAC protocol. UBPFCC is more efficient, and fairness of data dissemination in the vehicular network. This mechanism needs to context exchange among the neighbour nodes; however, it increased the overhead in the communication channel. To evaluating the message priority, depends on packet size and utility, therefore reducing the performance of dissemination of packet for event-driven safety message.

\section{1) Observations:}

Proactive congestion control schemes are very helpful to immediately evaluate the minimum-maximum rate of transmission. The proactive protocol is different from reactive protocol in two ways: first, rate evaluation is done independently and is controlled by the time, it changes flow for the network to register in the set of active flows such as arrival or departure flow which is itself equivalent to delay. Second, rates are evaluated specifically based on active flow, however, it avoiding adaptations that a reactive scheme needs to connect to target rates. The deficit of a calculation phase and smart/direct rate computations helps protocol quiet faster.

\section{Hybrid congestion control}

The third type of congestion control mechanism is hybrid congestion control. This approach depicts the virtue of reactive and proactive mechanisms. Example; adaption of the rate of messages is reactive and transmit power is proactive. However, it obtained by controlling packet rate generation, transmit power, the carrier sense threshold or a combination of transmission parameter. Some time it suffers from both hurdles (bandwidth overhead and latency), at the same time it reduces performance.

Due to the high density of traffic periodic beacons uses the entire bandwidth of medium, it results in congestion. However, the channel is not properly disseminated the packet. To solve the saturated condition Mughal et al. found mechanism, is relying on the concept of limitation of beacon bandwidth chunk for high priority even-driven message. To reducing the channel load, transmission rate and transmission power schemes are used. Zhang et al. purposed a new approach to reduce the congestion called as "concepts and framework congestion control" (CC-CF). CC-CF is a distributed in nature, whereas each node utilised both algorithm that is proactive and reactive. Event-driven and beacon message is control by reducing and a specific number of redundant packets. Guan et al. purposed the two-level adaptive message rate control (AMRC) algorithm. AMRC allowed the high availability channel for safety application. To find the favourable values of the messages rate, channel interval, quality of services and back-off exponent (BE) for the limited number of vehicles, an off-line procedure to be calculated at the first level. At the second level, roadside units (RSU) or access points are helpful to broadcast the acquired information to the vehicles. AMRC is performed like as centralized behaviour. The main motive of the AMRC scheme is trying to make the bandwidth free for non-safety and beacon messages. Fallah et at. Designed a novel channel occupancy-based congestion control (CO-CC) protocol. CO-CC focused to provide the congestion control in safety application at specific feedback from the vehicular network. It is results, making fast decisions on packet traffic condition CBT is using the metric in the channel. The main advantage of this approach is using the propagation model, transmission rate and vehicle density in the network.

Djahel et al. have introduced another congestion control scheme based on power and rate control (P\&PC). In this algorithm, has been three main key conditions that are priority assignment, beacon load adjustment and congestion detection. Also, congestion detection is using the three metrics; collision rate (CR), average waiting for time (AWT) and beacon reception rate (BRR). The power transmission and beacon generation rate is helpful to adjust the beacon load at fine-tuning. According to Le et al. for increasing road safety and traffic effectiveness, the intelligent transportation system (ITS) applications use the two types of messages: Beacon and event-driven messages. Here author evaluates three beacon congestion control algorithms whereas handling the beacon load under the predefined threshold by adapting the transmission power or transmission rate for the beacon messages. The distance between the receiver and the sender increases, the reception rates for both warning and beacon messages drop.

Huang et al. proposed an adaptive inter-vehicle communication control (AICC) mechanism. Here beacon changes both in a proactive way (generation rate) and in a reactive way (transmit power), to maintaining the channel load. For tuning the beacon generation rate and power two different congestion control approaches are applied for improving the vehicle's ability. Beacon generation rate is adjusted on the forecast (guess) tracking error of own position. Moreover, transmission power control is involved the observed the status of the channel, on the behalf of channel load. Hence both the beacons transmit power and generation rate uses the direction to control the parameters of transmission among the vehicles. Baldessari et al. are tried controlling the channel load. They designed novel schemes for the combination of message interval and power control into a one algorithm loop. The designed algorithm is based on the framework, which consists of three stages: load change estimation, channel monitoring and action. Whenever the channel usage is under the saturated level, the channel access time and reliability of the packet delivery reduce as the load increases.

\section{1) Observations:}

A hybrid congestion control aims to integrate the objectives of both reactive and proactive protocols, thus it combined the transmission rate and power for congestion control. Their results involve of enhanced rate control, enhanced power control and also joined rate and power control scheme. Channel load is calculated to obtain the number of immediate vehicles in the nearby area. To directly obtain the packet rate, evaluate the number of vehicles and predefined channel busy time threshold in a specific area However, no congestion control algorithm is appealed for the huge intersection, the transmit rates of both warning and beacon messages are lower-grade than those acquired with the congestion control protocol. 
Table1. Congestion control approaches and their different characteristics for vehicular networks:

\begin{tabular}{|c|c|c|c|c|c|c|c|c|c|}
\hline $\begin{array}{l}\text { Congestion } \\
\text { Control } \\
\text { Class }\end{array}$ & Approaches & $\begin{array}{l}\text { Access } \\
\text { Priority }\end{array}$ & Simulation & $\begin{array}{l}\text { Transmit } \\
\text { Power } \\
\text { Control }\end{array}$ & $\begin{array}{l}\text { Transmit } \\
\text { Data-Rate } \\
\text { Control }\end{array}$ & Performance & $\begin{array}{l}\text { Traffic } \\
\text { Density }\end{array}$ & CBT & Scenario \\
\hline \multirow{9}{*}{ PROACTIVE } & VCWC[21] & $x$ & Ns-2 & $x$ & $\checkmark$ & $\begin{array}{l}\text { Maximum delay (sec.), no. } \\
\text { of message, delay }\end{array}$ & $\begin{array}{l}\text { High } \\
\text { low }\end{array}$ & $x$ & Highway \\
\hline & UBPFC[1] & $x$ & Ns-2 & $x$ & $\checkmark$ & Drop-rate(bits/s) & High & $x$ & Highway \\
\hline & BRR-EPA[2] & $x$ & Ns-2 & $x$ & $\checkmark$ & Received packet ratio & High & $\checkmark$ & Highway \\
\hline & PULSAR[3] & $x$ & Ns-2 & $x$ & $\checkmark$ & Rate adaption & High & $x$ & Highway \\
\hline & D-FPAV [4] & $\sqrt{ }$ & Ns-2 & & $x$ & $\begin{array}{l}\text { Probability of message } \\
\text { reception, channel busy } \\
\text { time rate }\end{array}$ & High & $\sqrt{ }$ & Highway \\
\hline & $\begin{array}{l}\text { Application } \\
\text { Based } \\
\text { Congestion } \\
\text { Control [22] } \\
\end{array}$ & $x$ & Ns-2 & $\checkmark$ & $x$ & $\begin{array}{l}\text { Packet received/s, Trans- } \\
\text { mission power }(\mathrm{dBm})\end{array}$ & Low & $\checkmark$ & Highway \\
\hline & $\begin{array}{l}\text { UV-CAST } \\
{[5]}\end{array}$ & $\checkmark$ & SUMO & $x$ & $\checkmark$ & $\begin{array}{l}\text { Average received distance } \\
\text { (pkts), no. of messages }\end{array}$ & $\begin{array}{l}\text { High } \\
\text { Low }\end{array}$ & $x$ & $\begin{array}{l}\text { Highway } \\
\text { Urban }\end{array}$ \\
\hline & $\begin{array}{l}\text { MAC-CC } \\
{[6]}\end{array}$ & $x$ & MATLAB & $x$ & $\checkmark$ & $\begin{array}{l}\text { Throughput (Mbps), pack- } \\
\text { et-loss } \\
\text { probability }\end{array}$ & Low & $x$ & Urban \\
\hline & $\begin{array}{l}\text { Contextual C-CC } \\
{[7]}\end{array}$ & $\checkmark$ & Ns-2 & $x$ & $\checkmark$ & $\begin{array}{l}\text { Transmission power } \\
(\mathrm{dBm}), \mathrm{CBT}(\%)\end{array}$ & Low & $\checkmark$ & Highway \\
\hline \multirow{8}{*}{ REACTIVE } & DCC [23] & $x$ & Ns-2 & $\checkmark$ & $x$ & Average packet no. & High & $\checkmark$ & Highway \\
\hline & EMBARC [8] & $x$ & $\begin{array}{l}\text { Ns-2 } \\
\text { SUMO }\end{array}$ & $\checkmark$ & $\checkmark$ & $\begin{array}{l}\text { Tracking error }(\mathrm{m}) \text {, } \\
\text { Packet error ratio, } \\
\text { Mean IPG(sec.) }\end{array}$ & High & $\checkmark$ & Highway \\
\hline & PCCP [9] & $\checkmark$ & Ns-2 & $x$ & $\checkmark$ & Packet delivery ratio & Low & $\sqrt{ }$ & Highway \\
\hline & $\begin{array}{l}\text { Timeout } \\
\text { Mechanism } \\
{[10]}\end{array}$ & $x$ & Ns-2 & $x$ & $\checkmark$ & $\begin{array}{l}\text { Worst-case delay, no. of } \\
\text { missed deadline, latency } \\
\text { (clock, cycles),Delivery } \\
\text { throughput }\end{array}$ & Low & $x$ & Highway \\
\hline & TCP-Illinois [11] & $x$ & Ns-2 & $x$ & $\checkmark$ & $\begin{array}{l}\text { Throughput ratio b/w } \\
\text { measured throughput and } \\
\text { computed average } \\
\text { throughput }\end{array}$ & Low & $x$ & Highway \\
\hline & $\begin{array}{l}\text { Combined-CC } \\
{[12]}\end{array}$ & $\checkmark$ & $\begin{array}{l}\text { PML, } \\
\text { PSMR }\end{array}$ & $x$ & $\checkmark$ & Delay-received (in sec.) & Low & $x$ & Highway \\
\hline & $\begin{array}{l}\text { Markov Chain } \\
{[13]}\end{array}$ & $\checkmark$ & Ns-2 & $\checkmark$ & $\checkmark$ & Loss rate $(\%)$ & Low & $x$ & Highway \\
\hline & $\begin{array}{l}\text { Power \& Rate } \\
\text { combined CC } \\
{[14]}\end{array}$ & $\checkmark$ & Ns-2 & $\sqrt{ }$ & $\checkmark$ & Packet delivery ratio & Low & $x$ & Highway \\
\hline \multirow{6}{*}{ Hybrid } & CC-CF [15] & $x$ & Ns-2 & $\checkmark$ & $\checkmark$ & Network performance & High & $x$ & Highway \\
\hline & AMRC [16] & $\checkmark$ & Ns-2 & $x$ & & $\begin{array}{l}\text { Average packet delay, } \\
\text { message success probabil- } \\
\text { ity }\end{array}$ & Low & $x$ & Highway \\
\hline & CO-CC [17] & $x$ & $\begin{array}{l}\text { OPNET, } \\
\text { SHIFT, } \\
\text { NS-2 } \\
\end{array}$ & $\sqrt{ }$ & $x$ & $\begin{array}{l}\text { Channel occupancy IDR } \\
\text { (pkt nodes/sec) }\end{array}$ & High & $\checkmark$ & Highway \\
\hline & P \& RC [18] & $\checkmark$ & OPNET & $x$ & $\checkmark$ & $\begin{array}{l}\text { Beacon delivery ratio,tota } \\
\text { delay }(\mathrm{ms}) \text {, emergency } \\
\text { message reception ratio }\end{array}$ & Low & $\checkmark$ & Highway \\
\hline & $\begin{array}{l}\text { Beacon } \\
\text { Congestion } \\
\text { Control [19] } \\
\end{array}$ & $\checkmark$ & Ns-2 & $\checkmark$ & $\checkmark$ & $\begin{array}{l}\text { Reception rate }(\%) \\
\text { CBT }(\%)\end{array}$ & High & $\checkmark$ & Highway \\
\hline & AICC [20] & $x$ & OPNET & $\checkmark$ & $\checkmark$ & $\begin{array}{l}\text { Population of tracking } \\
\text { error }\end{array}$ & High & $x$ & Highway \\
\hline
\end{tabular}




\section{CONLCUTION}

Congestion has been involved as a key challenge in vehicular communication. The limited or narrow bandwidth in vehicular network is a main cause of congestion. In this paper, we describe the basic of VANET architecture and communication channel for various application of vehicular network in ITS. This paper is focused on congestion at MAC layer and analyzes how to control the congestion problem during the vehicle's communication. In ITS, an congestion control is essential for maintaining the vehicle safety on the road. Various congestion control scheme under varying scenarios and topology are elaborated with their pros and cons. The literature survey is provided by analyzing the effect of transmit power control, packet generation rate, access priority, utility function and carrier sense threshold to control the congestion. Using efficient congestion control mechanism, chances of road accident may get reduced. Therefore, future research can be preceded by looking on the gaps in the existing studies.

\section{ACKNOWLEDGMENT}

I would like to thank the department of computer and information security of Punjab engineering college, Chandigarh. I am very thankful to my corresponding author Mrs. Swati Sharma for her guidance and encouraging discussion on congestion control in a network.

\section{REFERENCES}

[1] L Wischhof, H Rohling. Congestion control in vehicular ad hoc networks. InIEEE International Conference on Vehicular Electronics and Safety, 2005. 2005 Oct 14 (pp. 58-63). IEEE.

[2] M Torrent-Moreno, D Jiang, H Hartenstein. Broadcast reception rates and effects of priority access in 802.11-based vehicular ad-hoc networks. InProceedings of the 1st ACM international workshop on Vehicular ad hoc networks 2004 Oct 1 (pp. 10-18). ACM.

[3] T Tielert, D Jiang, Q Chen, L Delgrossi, H Hartenstein. Design methodology and evaluation of rate adaptation based congestion control fsor vehicle safety communications. In2011 IEEE Vehicular Networking Conference (VNC) 2011 Nov 14 (pp. 116-123). IEEE.

[4] M Torrent-Moreno, P Santi, H Hartenstein. Distributed fair transmit power adjustment for vehicular ad hoc networks. In2006 3rd Annual IEEE Communications Society on Sensor and Ad Hoc Communications and Networks 2006 Sep 28 (Vol. 2, pp. 479-488).IEEE.

[5] W Viriyasitavat, OK Tonguz, F Bai. UV-CAST: an urban vehicular broadcast protocol. IEEE Communications Magazine. 2011 Nov;49(11):116-24.

[6] CW Hsu, CH Hsu, HR Tseng. MAC channel congestion control mechanism in IEEE 802.11 p/WAVE vehicle networks. In2011 IEEE Vehicular Technology Conference (VTC Fall) 2011 Sep 5 (pp. 1-5). IEEE.

[7] M Sepulcre, J Gozalvez, J Harri, H Hartenstein. Contextual communications congestion control for cooperative vehicular networks. IEEE Transactions on Wireless Communications. 2011 Feb;10(2):385-9.

[8] C Wang, K Sohraby, V Lawrence, B Li, Y Hu. Priority-based congestion control in wireless sensor networks.In IEEE International Conference on Sensor Networks, Ubiquitous, and Trustworthy Computing (SUTC'06) 2006 Jun 5 (Vol. 1, pp. 8-pp).IEEE.

[9] G Bansal, H Lu, JB Kenney, C Poellabauer. EMBARC: Error model based adaptive rate control for vehicle-to-vehicle communications. InProceeding of the tenth ACM

[10] S Liu, T Başar, R Srikant. TCP-Illinois: A loss-and delay-based congestion control algorithm for high-speed networks. Performance Evaluation. 2008 Jun 1;65(6-7):417-40.
[11] AH Smai, LE Thorelli.Global reactive congestion control in multicomputer networks.InProceedings.Fifth International Conference on High Performance Computing (Cat. No. 98EX238) 1998 Dec 17 (pp. 179186). IEEE.

[12] A Mondal, S Mitra. Dynamic and distributed channel congestion control strategy in VANET. In2014 International Conference on Advances in Computing, Communications and Informatics (ICACCI) 2014 Sep 24 (pp. 1697-1703).IEEE.

[13] MA Benatia, L Khoukhi, M Esseghir, LM Boulahia. A Markov chain based model for congestion control in VANETs. In2013 27th International Conference on Advanced Information Networking and Applications Workshops 2013 Mar 25 (pp. 1021-1026). IEEE.

[14] BM Mughal, AA Wagan, H Hasbullah. Efficient congestion control in VANET for safety messaging.In2010 International Symposium on Information Technology 2010 Jun 15 (Vol. 2, pp. 654-659).IEEE.

[15] W Zhang, A Festag, R Baldessari, L Le. Congestion control for safety messages in VANETs: Concepts and framework. In2008 8th International Conference on ITS Telecommunications 2008 Oct 24 (pp. 199203). IEEE.

[16] W Guan, J He, C Ma, Z Tang, Y Li. Adaptive message rate control of infrastructured DSRC vehicle networks for coexisting road safety and non-safety applications. International Journal of Distributed Sensor Networks. 2012 Aug 16;8(8):134238.

[17] YP Fallah, C Huang, R Sengupta, H Krishnan. Congestion control based on channel occupancy in vehicular broadcast networks. In2010 IEEE 72nd Vehicular Technology Conference-Fall 2010 Sep 6 (pp. 15). IEEE.

[18] S Djahel, Y Ghamri-Doudane. A robust congestion control scheme for fast and reliable dissemination of safety messages in VANETs. In2012 IEEE Wireless Communications and Networking Conference (WCNC) 2012 Apr 1 (pp. 2264-2269). IEEE.

[19] L Le, R Baldessari, P Salvador, A Festag, W Zhang. Performance evalusation of beacon congestion control algorithms for VANETs. In2011 IEEE Global Telecommunications Conference-GLOBECOM 20112011 Dec 5 (pp. 1-6). IEEE.

[20] CL Huang, YP Fallah, R Sengupta, H Krishnan. Adaptive intervehicle communication control for cooperative safety systems. IEEE network. 2010 Jan;24(1):6-13.

[21] X Yang, L Liu, NH Vaidya, F Zhao.A vehicle-to-vehicle communication protocol for cooperative collision warning.InThe First Annual International Conference on Mobile and Ubiquitous Systems: Networking and Services, 2004. MOBIQUITOUS 2004. 2004 Aug 22 (pp. 114123). IEEE.

[22] M Sepulcre, J Gozalvez, J Harri, H Hartenstein. Application-based congestion control policy for the communication channel in VANETs. IEEE Communications Letters. 2010 Oct;14(10):951-3.

[23] S Subramanian, M Werner, S Liu, J Jose, R Lupoaie, X Wu. Congestion control for vehicular safety: synchronous and asynchronous MAC algorithms. InProceedings of the ninth ACM international workshop on Vehicular inter-networking, systems, and applications 2012 Jun 25 (pp. 63-72).ACM. 